\title{
MEASURING A BINARY'S ORIENTATION WITH LISA
}

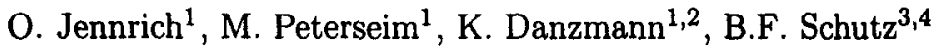

${ }^{1}$ Institut für Atom- und Molekülphysik, Universität Hannover, Appelstr. 2, 30167 Hannover, Germany

${ }^{2}$ Max-Planck-Institut für Quantenoptik, Hans-Kopfermann-Str. 1, 85748 Garching, Germany

${ }^{9}$ University of Wales, College of Cardiff, UK

4 Albert-Einstein-Institut, Max-Planck-Institut für Gravitationsphysik, Potsdam, Germany

\section{ABSTRACT}

We are presenting numerical results concerning LISA's ability to distinguish between different polarizational states of a gravitational wave. Therefore, we assume a binary as a source of a gravitational wave, finding its orientation which determines the polarization of the gravitational wave. By means of signal processing, we are able to give the $1 \sigma$-uncertainty for determining the orientation of the source. $(\odot 2003$ Published by Elsevier Ltd on behalf of COSPAR.

\section{INTRODUCTION}

The most promising sources of gravitational waves within the frequency range of $0.1 \ldots 100 \mathrm{mHz}$ (Danzmann et al., 1996) are neutron star binaries. Emitting in this frequency interval their dynamics are mainly underlying Newtonian mechanics, leading to a frequency of the emitted gravitational wave being constant over typical timescales of a measurement performed by LISA (1-2 years). Among the parameters of a gravitational wave the polarizational state reveals information about the orientation of a binary.

\section{SIGNAL FROM A BINARY}

Consider two point-like masses $m_{1}$ and $m_{2}$, forming a binary and moving in a circular orbit. Under the above assumption of low frequency, the emitted gravitational wave will be monochromatic in the source's reference frame, so that for such a system, having total mass $M=m_{1}+m_{2}$ and reduced mass $\mu=m_{1} m_{2} / M$, the tensor $\mathbf{h}^{t t}$ representing the gravitational wave, is of the form (Thorne, 1989)

$$
\mathbf{h}^{t t}=\frac{4 \mu}{R}(M \omega)^{\frac{2}{3}} \Re\left\{\left(\begin{array}{cc}
h_{+} & h_{\times} \\
h_{\times} & -h_{+}
\end{array}\right) \exp [2 \mathrm{i} \omega(z / c-t)]\right\}
$$

with the amplitudes $h_{+}$and $h_{\mathrm{x}}$ of the plus- and cross-polarization, respectively.

To cover different orientations of the binary we have introduced usual spherical coordinates ( $\cos \beta, \alpha)$ for the angular momentum of the binary, $\beta$ denoting the angle enclosed by the angular momentum and the $z$-axis, $\alpha$ denoting the angle enclosed by the projection of the angular momentum onto the $x$-y-plane and the $x$-axis. 
This leads to a gravitational wave propagating along the $z$-axis with amplitudes

$$
\begin{aligned}
& h_{+}=\frac{1}{2}\left(\cos ^{2} \beta+1\right) \cos 2 \alpha+\mathrm{i} \cos \beta \sin 2 \alpha \\
& h_{\mathrm{x}}=\frac{1}{2}\left(\cos ^{2} \beta+1\right) \sin 2 \alpha-\mathrm{i} \cos \beta \cos 2 \alpha
\end{aligned}
$$

Choosing the angular momentum aligned with the $z$-axis $(\beta=0)$ leads to a circularly polarized gravitational wave, whereas aligning the angular momentum with the $x$-axis or the bisector of the $x$-axis and $y$-axis $\left(\beta=\frac{\pi}{2}, \alpha=0\right.$ or $\alpha=\frac{\pi}{4}$ ) gives a linearly plus polarized or a linearly cross polarized gravitational wave, respectively.

\section{GAUSSIAN APPROXIMATION}

To obtain results concerning LISA's ability to distinguish different orientations of a binary, we made use of methods of signal processing and extraction presented by Cutler and Flanagan (1994). Given a real signal $s(t ; \vec{\mu})=m(t ; \vec{\mu})+n(t)$ obtained by the detector, with $m(t ; \vec{\mu})$ denoting the noise-free signal and $n(t)$ symbolizing the noise, the probability $P(\vec{\mu} \mid s) \mathrm{d} \vec{\mu}$ to find a parametrization within the interval $[\vec{\mu}, \vec{\mu}+\mathrm{d} \vec{\mu}]$ for the signal $s(t ; \vec{\mu})$ is Gaussian provided sufficiently high signal-to-noise ratios $\rho$ (Finn, 1992). Given a constant probability for all degrees of freedom, i.e. components of the vector $\vec{\mu}$, the probability $P(\vec{\mu} \mid s) \mathrm{d} \vec{\mu}$ is centered around the parametrization $\vec{\mu}_{0}$ for the noise-free signal $m\left(t, \vec{\mu}_{0}\right)$. The covariance matrix $\sigma_{i j}^{2}$ is then given by

$$
\sigma_{i j}^{2}=C_{i j}
$$

The matrix $\mathbf{C}^{-1}$ is called the Fisher-matrix and is defined by means of the inner product of the derivatives of the signal.

$$
C_{i j}^{-1}=2\left\langle\partial_{i} s(t ; \vec{\mu}), \partial_{j} s(t ; \vec{\mu})\right\rangle
$$

The shorthand notation $\partial_{i}$ denotes the derivative with respect to the $i$-th component of the vector $\vec{\mu}$.

The inner product of two functions is for these purposes defined as a convolution weighted with the expected spectral density $\mathcal{S}_{\mathrm{n}}(f)$ of the noise (Gürsel and Tinto, 1989) performed in Fourier space.

$$
\langle a, b\rangle=\int_{0}^{\infty} \mathrm{d} f \frac{\tilde{a}(f) \tilde{b}^{*}(f)+\tilde{a}^{*}(f) \tilde{b}(f)}{\mathcal{S}_{\mathbf{n}}(f)}
$$

The relation of the quadratic spectral density of the noise $\mathcal{S}_{\mathfrak{n}}(f)$ and the $\rho$ is given by Finn (1992) and Thorne (1989)

$$
\rho^{2} \approx 2\langle m(\vec{\mu}), m(\vec{\mu})\rangle
$$

Having a signal which is concentrated on a small frequency interval, $\mathcal{S}_{\mathbf{n}}(f)$ can be considered as constant over this frequency interval. Thus Eq. (5) can be written as

$$
C_{i j}^{-1}=\rho^{2} \frac{\int_{0}^{\infty} \mathrm{d} f \partial_{i} \tilde{m} \partial_{j} \tilde{m}^{*}+\partial_{i} \tilde{m}^{*} \partial_{j} \tilde{m}}{\int_{0}^{\infty} \mathrm{d} f|\tilde{m}|^{2}}
$$

The 'error volume' $\Sigma$ is given by the determinant of the covariance matrix,

$$
\Sigma=\sqrt{\operatorname{det} \mathbf{C}}=\frac{1}{\sqrt{\operatorname{det} C^{-1}}}
$$




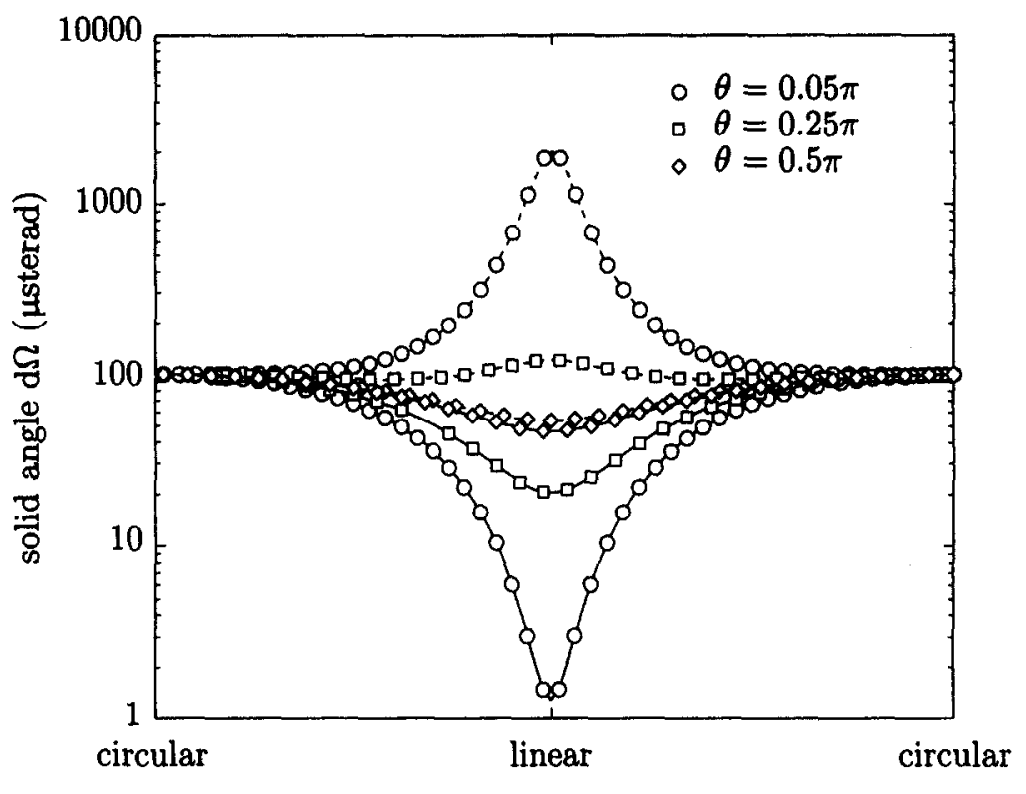

Polarizational state

Fig. 1. Maximum (dashed lines) and minimum (solid curves) errors of orientation for various source positions.

thus, from Eq. (8) it is easily obtained that

$$
\Sigma \sim \rho^{-n}
$$

$n$ being the dimension of $\vec{\mu}$, hence the dimension of the parameter space.

\section{APPLICATION TO POLARIZATION}

In the case of the angular momentum of a binary the vector $\vec{\mu}$ consists of the two entries $(\cos \beta, \alpha)$ and the 'error volume' $\Sigma$ is usually denoted as the solid angle $\mathrm{d} \Omega$

$$
\Sigma=\mathrm{d} \Omega=\mathrm{d} \alpha \mathrm{d} \beta \sin \beta
$$

supposing $\alpha$ and $\cos \beta$ being uncorrelated. The meaning of $d \Omega$ is the following: Given a unit vector $\vec{n}$ pointing along the angular momentum of the binary, the top of $\vec{n}$ will move within the area $\mathrm{d} \Omega$ while tilting the plane of rotation of the binary a bit. Hence, $d \Omega$ is a measure for the accuracy with which the orientation of the binary can be detected.

\section{RESULTS}

All the results presented in the following are obtained by using a fixed signal-to-noise ratio $\rho=100$ as it seems to be reasonable for signals expected in the frequency range around $3 \mathrm{mHz}$. Moreover, we restricted ourselves to a monochromatic source with a frequency $f_{\mathrm{g}}$ of $3 \mathrm{mHz}$. Figure 1 shows the orientation error as a function of the polarization state for various source positions. The maximum and minimum curves belong to different values of $\alpha$, hence different initial phases. Whereas the solid angle is almost around $100 \mu \mathrm{sr}$ for circularly polarized gravitational waves, it varies by three orders of magnitude for linearly polarized gravitational waves for sources close to the poles.

LISA is at certain times insensitive to a given linear polarization emitted by sources close to or at the poles. Even for a one-year integration time this leads to a considerable increase in uncertainty. In contrast, such zeros in the sensitivity do not exist for circularly polarized gravitational waves, leading to the observed insensitivity to the source's position. 


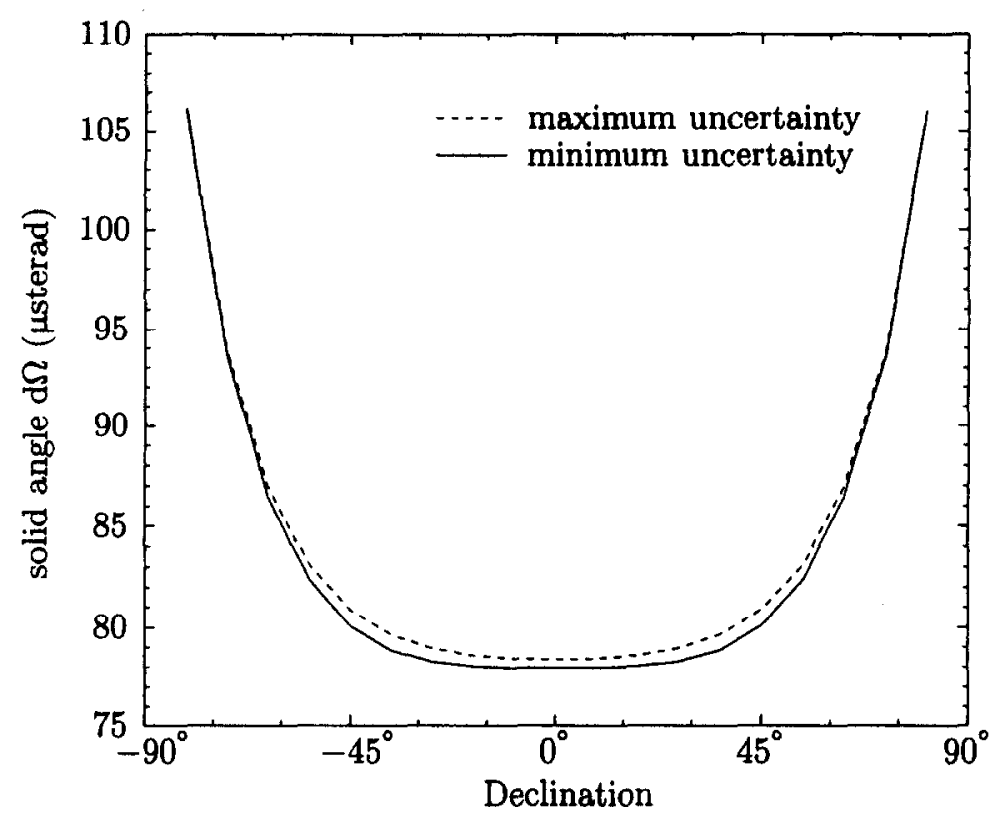

Fig. 2. Averaged error of orientation as function of source declination. Variations with the azimuth are small compared to overall variations.

To obtain a result independent from the binary's orientation, we averaged over every possible orientation. Therefore, Figure 2 gives the averaged error of orientation as a function of the declination of the source, $0^{\circ}$ denoting the ecliptic, $\pm 90^{\circ}$ the poles.

The averaged error of orientation increases towards the pole, clearly an effect of the poor resolution for linearly polarized gravitational waves described above. However, the variations with the azimuth of the source are small compared to the overall variations, giving a distribution close to rotational symmetry. It should be noted that in contrast to the results obtained by (Peterseim et al., 1996) for the angular resolution, the resolution for the orientation of a binary is best for sources located in the ecliptic, decreasing towards the poles.

\section{CONCLUSION}

Using the method of Gaussian approximation, we obtained $110 \mu \mathrm{sr}$ at a signal-to-noise ratio of 100 as an upper limit for the uncertainty of the orientation of a binary observed by LISA. Although restricted to monochromatic binaries at a frequency of $3 \mathrm{mHz}$ with circular orbits, it is clear that this result will encourage the use of LISA as an astronomers' instrument.

\section{REFERENCES}

Cutler, C., and E.E. Flanagan, Phys. Rev. D, 49, 2658-2697, 1994.

Danzmann, K., J. Hough, P. Bender, R.W.. Hellings, R. Schilling, A. Rüdiger, B.F. Schutz, and T. Sumner, LISA Pre-Phase A Report, MPQ-Report 208, Max-Planck Institut für Quantenoptik, Garching, 1996.

Finn, L.S., Phys. Rev. D, 46, 5236-5249, 1992.

Gürsel, Y., and M. Tinto, Phys. Rev. D, 40, 3885-3995, 1989.

Peterseim, M., O. Jennrich, K. Danzmann, and B.F. Schutz, Class. Quantum Grav., Proc. on LISASymposium, 1996.

Thome, K., in 300 Years of Gravitation, ed. S.W. Hawking and W. Israel, pp. 330-458, Cambridge University Press, 1989. 